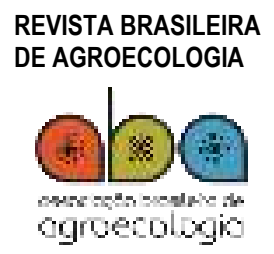

ISSN: $1980-9735$

DOI: $10.33240 /$ rba.v15i5.23236

Vol. 15 | No 5 | p. $213-221 \mid 2020$

ARTIGO

\title{
LEVANTAMENTO FITOSSOCIOLÓGICO DE PLANTAS ESPONTÂNEAS EM CORREDORES AGROECOLÓGICOS
}

\author{
Phytosociological survey of weeds in agroecological corridors
}

\author{
Wellington Pereira de Carvalho ${ }^{1}$ e Cynthia Torres de Toledo Machado²
}

\section{RESUMO}

O estudo foi realizado em duas propriedades situadas nos municípios de Paracatu (MG) e Silvânia (GO), manejadas sob sistema orgânico, que fazem parte do projeto "Corredores agroecológicos como estratégias para a produção de alimentos e sementes, focados no manejo da agrobiodiversidade e sustentabilidade de pequenas propriedades familiares" entre os anos de 2016 a 2019. Seu objetivo foi fazer o levantamento fitossociológico da composição da vegetação espontânea nos corredores agroecológicos, obtendo dados de frequência, densidade e índice de valor de cobertura das espécies ocorrentes naquela formação, além de realizar estudo das semelhanças e diferenças de comunidades espontâneas entre as áreas avaliadas, através de coeficiente de similaridade. No total, foram identificadas 29 espécies, sendo 10 presentes em ambas as regiões. Asteraceae e Poaceae foram as duas principais famílias, entre as 16 encontradas. O coeficiente de similaridade entre as duas regiões foi de 57\%, mostrando similaridade mediana entre a flora nos dois anos estudados. A espécie mais importante em ambas as propriedades é Pennisetum setosum.

Palavras-chave: Pennisetum setosum. Agricultura Orgânica. Análise Fitossociológica. Plantas Daninhas.

\section{ABSTRACT}

The study was carried out in two properties located in the municipalities of Paracatu (MG) and Silvânia (GO), managed under an organic system that are part of the project "Agroecological corridors as strategies for the production of food and seeds, focused on the management of agrobiodiversity and sustainability of small family properties" between the years of 2016 to 2018. Its objective was to carry out a phytosociological survey of the composition of spontaneous vegetation in agroecological corridors, obtaining data on frequency, density, abundance and index of relative importance of the species occurring in that formation, in addition to conducting a study of the similarities and differences of spontaneous communities between the evaluated areas, through similarity coefficient. In total, 29 species were identified, 10 of which were present in both regions. Asteraceae and Poaceae were the two main families, among the 16 found. The similarity coefficient between the two regions was $57 \%$ for cynthia.torres@embrapa.br

Recebido em: 11/05/2020 Aceito para publicação em: 08/09/2020 the years of 2017 and 2018, showing an average similarity in the
most important specie in both properties is Pennisetum setosum.

Correspondência para: wellington.carvalho@embrapa.br 
Introdução

A presença de plantas espontâneas nas culturas cultivadas em sistemas agroecológicos pode ser discutida sob dois diferentes aspectos. Do ponto de vista apenas da produção, a infestação de plantas espontâneas representa problema de difícil solução para a agricultura por causa do número de espécies invasoras, que emergem em épocas diferentes e competem de forma diferenciada com as culturas agrícolas, interferindo, sobremaneira, na produtividade das plantas cultivadas e na operacionalização dos sistemas de produção. Através da competição por água, luz e nutrientes minerais, de ações indiretas, como a hospedagem e a transmissão de pragas e doenças e, muitas vezes, de ações alelopáticas, as plantas espontâneas ocasionam perdas na produção das culturas. Por outro lado, analisando o sistema agrícola, as plantas espontâneas nas culturas podem trazer benefícios como atuar como hospedeiras de inimigos naturais, promover a cobertura do solo, servir de fonte de nutrientes (ciclagem) e matéria orgânica, melhorar as propriedades físico-químicas dos solos, entre outros (CHAVES e CALEGARI, 2001).

A cobertura vegetal de determinada área resulta de causas atuais, como manejo, clima, solo e ação da fauna. Os indivíduos da mesma espécie (que podem reagir diferentemente a essas causas) compõem uma população, e grupos de populações que ocorrem juntas caracterizam uma comunidade. As comunidades podem diferenciar-se, dependendo das interações das espécies com o meio abiótico (OLIVEIRA e FREITAS, 2008). Uma vez que as comunidades podem variar sua composição florística em função do tipo de manejo aplicado à área, o reconhecimento das espécies presentes torna-se fundamental, quanto mais se for levado em conta o custo financeiro e ambiental de seu controle (ERASMO et al, 2004).

Dessa maneira, é importante investir em métodos que auxiliem no conhecimento dessas comunidades. Um dos métodos mais utilizados no reconhecimento florístico em áreas agrícolas ou não é o denominado estudo fitossociológico, que pode ser conceituado como "a ecologia da comunidade vegetal e envolve as inter-relações de espécies vegetais no espaço e, de certo modo, no tempo" (ERASMO et al., 2004). A aplicação de um método fitossociológico ou quantitativo num dado local e num dado tempo permite fazer uma avaliação da composição da vegetação, obtendo dados de frequência, densidade, índice de valor de cobertura e coeficiente de similaridade das espécies ocorrentes naquela formação (BRAUN-BLANQUET, 1979). Assim, o método fitossociológico é uma ferramenta que, se usada adequadamente, permite fazer várias inferências sobre a comunidade em questão (ERASMO et al., 2004).

No Brasil, estudos fitossociológicos foram realizados para diversas culturas, como o girassol (ADEGAS et al., 2010), aveia e trigo (COSTA et al., 2014), pastagens (FERREIRA et al., 2014), mandioca (CARDOSO et al., 2013), cana-de-açúcar (SILVA et al., 2017), feijão (TAVARES et al., 2013). Em sistemas orgânicos, alguns estudos como o de Maciel et al. (2010) na cultura do café e Melo et al. (2007) na cultura do milho, evidenciam a importância de se proceder a levantamentos da flora espontânea como instrumento de auxílio ao seu controle.

Os "corredores agroecológicos" são faixas contínuas e intercaladas de diferentes cultivos alimentares e de diferentes espécies de cobertura, planejadas de modo que sejam rotacionadas no tempo e manejadas seguindo as premissas da produção de base agroecológica (MACHADO e MACHADO, 2015). A estratégia representa a síntese do manejo da agrobiodiversidade, em que o corredor combina consórcios e sucessões de espécies de interesse dos agricultores utilizando as melhores variedades das espécies escolhidas. As variedades/genótipos mais adaptados são definidos a partir de ensaios de competição e seleção prévios, bem como de unidades de demonstração, escolhidos pela produtividade ou características específicas e cultivadas em faixas (MACHADO e MACHADO, 2015).

O corredor possui por finalidade a produção de alimentos, representando, também, uma metodologia alternativa para a produção de sementes em nível comunitário ou de propriedade, além de promover a diversificação de cultivos e a melhoria nas condições de fertilidade dos solos e no manejo de pragas e doenças (MACHADO e MACHADO, 2015). 
Na estrutura dos corredores, a composição das faixas alterna cultivos alimentares com espécies de adubos verdes, plantas de cobertura, ou outras como aromáticas e condimentares que possam atuar como repelente, além de ter uso alimentar e valor comercial, como o gergelim. Essa estrutura é alterada nos plantios subsequentes, no ano seguinte, com a inversão das faixas: as de cultivos alimentares são substituídas por faixas de plantas recuperadoras da fertilidade, como os adubos verdes. Os corredores devem ser próximos ou limitados por vegetação nativa em pelo menos um dos lados, de modo que esta sirva de abrigo para a fauna benéfica e potencialize a população de inimigos naturais e o controle biológico de pragas (MACHADO e MACHADO, 2015).

Com este trabalho, objetivou-se identificar e quantificar as principais plantas espontâneas presentes nas áreas de corredores agroecológicos, por meio de levantamento fitossociológico realizado em fazendas orgânicas nas cidades de Silvânia e Catalão no estado de Goiás.

\section{Metodologia}

O estudo foi conduzido em duas propriedades rurais típicas de Agricultura Familiar, já convertidas ao sistema orgânico de produção. Essas áreas fazem parte do projeto "Corredores agroecológicos como estratégias para a produção de alimentos e sementes, focados no manejo da agrobiodiversidade e sustentabilidade de pequenas propriedades familiares". O objetivo geral desse projeto é a validação da estratégia dos corredores agroecológicos como sistema de produção para pequenos agricultores familiares de Goiás, tendo como culturas principais o milho, o feijão e a mandioca. A partir de estudos sobre o funcionamento desse sistema na adaptação de genótipos, foram analisadas variáveis químicas, físicas e biológicas de solo, ocorrência de insetos-praga, inimigos naturais, plantas espontâneas e aspectos socioeconômicos.

A Fazenda Piracanjuba localiza-se no município de Silvânia (GO), que está a, aproximadamente, 85 km da capital Goiânia. A sede da propriedade localiza-se a 163' $59,7^{\prime \prime}$ de latitude sul e 4835'52,8" de longitude oeste, estando a $853 \mathrm{~m}$ de altitude. O clima da região é do tipo Aw, segundo a classificação climática de Köppen, com inverno (maio a setembro) seco e verão (outubro a abril) chuvoso. Na porção do município com essas características de clima e altitude tendem a predominar os latossolos vermelhos e os vermelhos amarelos (BLANCANEAUX et al., 2007). As avaliações foram realizadas em novembro de 2016, dezembro de 2017 e novembro de 2018. A área avaliada foi de $900 \mathrm{~m}^{2}$.

A Fazenda Corinalves situa-se no distrito de Santo Antônio do Rio Verde, município de Catalão (GO), distando de $322 \mathrm{~km}$ de Goiânia. A propriedade localiza-se a 17055'21,6" de latitude sul e $47034^{\prime} 28,1^{\prime \prime}$ de longitude oeste, estando a, aproximadamente, $873 \mathrm{~m}$ de altitude. O clima é classificado como tropical de altitude (classificação climática de Köppen), com duas estações bem definidas, uma seca de junho a setembro, normalmente, e uma chuvosa, de dezembro a março. A pluviosidade média é estimada em 1.300mm (IMBEES, 2016). Predominam, no município de Catalão, os Latossolos Vermelho, Vermelho-Amarelo e os Cambissolos (RESENDE, 2016), muito provavelmente essas classes estão representadas nas diferentes porções da Fazenda Corinalves. As avaliações foram realizadas no mês de dezembro de 2017, em novembro de 2018 e dezembro de 2019. A área avaliada foi de $2.550 \mathrm{~m}^{2}$.

Para as duas áreas estudadas, o preparo do solo, faz-se comumente com uma gradagem por ano e o controle de plantas espontâneas se dá por roçadeira ou enxada. Após as colheitas, em todos os anos, as áreas são mantidas em pousio.

Para caracterização e estudo fitossociológico nas duas propriedades foi utilizado o método quadrado inventário e foi empregado como unidade amostral um quadro $(0,5 \times 0,5 \mathrm{~m})$, lançando-se 10 quadros aleatoriamente dentro de cada área de estudo, por meio de um caminhamento em ziguezague (ERASMO et al., 2004). As amostragens foram realizadas antes da gradagem para instalação das culturas que fazem parte dos corredores agroecológicos. Em cada quadro amostrado as plantas foram identificadas segundo a família, o gênero e a espécie, bem como foi feita a determinação do número presente de cada espécie. Na identificação e quantificação das espécies por nome científico, nome 
popular e família, foi utilizada literatura presente em Lorenzi (2008), com auxílio nas delimitações das famílias e ordenamento de alguns gêneros, em Souza e Lorenzi (2005).

Além da quantificação das espécies e do total dos indivíduos por área amostrada, foram, ainda, calculados os seguintes parâmetros fitossociológicos propostos por Mueller-Dombois e Ellenberg (1974):

Frequência $=$ no de quadrados onde a espécie foi encontrada $/$ no total de quadrados

Frequência relativa $=$ frequência da espécie $\times 100 /$ frequência total das espécies

Densidade $=$ no total de indivíduos da espécie) $/$ no total de quadrados

Densidade relativa $=$ densidade da espécie $\times 100 /$ densidade total das espécies

( $n$ o total de indivíduos amostrados)

Índice de Valor de Cobertura (IVC) = frequência relativa + densidade relativa

o Coeficiente de Similaridade (CS) foi calculado baseando-se na fórmula proposta por Sorensen (1972):

$C S=\{(2 \times n \circ$ de espécies comuns aos dois habitats $) \times 100 /$ ( $\mathrm{n}$ - de espécies do habitat $A+n$ o de espécies do habitat B)\}

O CS varia de 0 a 100, sendo máximo quando todas as espécies são comuns às duas áreas e mínimo quando não existem espécies em comum.

A frequência mostra o quanto agregada uma espécie está, ou não, na amostragem e, para isso, tem que ser examinada em conjunto com a densidade. Números de densidade altos com baixa frequência indicam agregação, o oposto, dispersão. A densidade é o número de indivíduos de cada espécie que compõe uma comunidade vegetal por unidade de superfície, sendo expressa em plantas $\mathrm{m}^{-}$ ${ }^{2}$. O índice de valor de cobertura é a junção de todas as avaliações realizadas, dando uma análise geral de qual espécie tem maior relevância na área amostrada.

\section{Resultados e discussão}

Nas duas áreas estudadas, pôde-se observar maior ocorrência de plantas dicotiledôneas, (70\% na fazenda Piracanjuba e 75\% na fazenda Corinalves). Na fazenda Piracanjuba, foram identificadas 24 espécies distribuídas em 10 famílias (Tabela 1 ). 
Tabela 1. Relação de famílias, espécies e seus nomes populares identificadas (X) nos levantamentos realizados nos anos de 2016 a 2019, nas fazendas Piracanjuba (P), situada no município de Silvânia (GO) e Corinalves (C), situada no município de Catalão (GO).

\begin{tabular}{|c|c|c|c|c|}
\hline \multirow[t]{2}{*}{ Família } & \multirow[t]{2}{*}{ Espécie } & \multirow[t]{2}{*}{ Nome popular } & \multicolumn{2}{|c|}{ Local } \\
\hline & & & $\mathrm{P}$ & C \\
\hline Asteraceae & Ageratum conyzoides & Mentrasto & $x$ & $X$ \\
\hline Amarantaceae & Alternanthera tenella & Apaga fogo & $\mathrm{X}$ & $\mathrm{X}$ \\
\hline Amarantaceae & Amaranthus deflexus & Caruru & $x$ & $X$ \\
\hline Asteraceae & Bidens pilosa & Picão preto & $x$ & - \\
\hline Asteraceae & Blainvillea rhomboidea & Picão grande & - & $\mathrm{X}$ \\
\hline Asteraceae & Conyza bonariensis & Buva & $x$ & - \\
\hline Asteraceae & Emilia fosbergii & Falsa serralha & $x$ & $\mathrm{X}$ \\
\hline Asteraceae & Melampodium perfoliatum & Estrelinha & $x$ & - \\
\hline Asteraceae & Tridax procumbens & Erva de Touro & $x$ & - \\
\hline Commelinaceae & Commelina benghalensis & Trapoeraba & $x$ & $\mathrm{X}$ \\
\hline Convolvulaceae & Ipomea triloba & Corda de viola & $x$ & $\mathrm{X}$ \\
\hline Cyperaeae & Cyperus rotundus & Tiririca & $x$ & $\mathrm{X}$ \\
\hline Euphorbiaceae & Chamaesyce hirta & Erva de Santa Luzia & $x$ & - \\
\hline Euphorbiaceae & Euphorbia heterophylla & Leiteira & $x$ & - \\
\hline Euphorbiaceae & Ricinus communis & Mamona & $x$ & - \\
\hline Euphorbiaceae & Sebastiana corniculata & Falsa guanxuma & - & $X$ \\
\hline Fabaceae & Senna ocidentalis & Fedegoso & - & $X$ \\
\hline Lamiaceae & Hyptis suaveolens & Cheirosa & $x$ & - \\
\hline Malvaceae & Sida cordifolia & Vassourinha & $x$ & - \\
\hline Malvaceae & Sida glaziovii & Vassourinha & $x$ & $X$ \\
\hline Malvaceae & Sida rhombifolia & Vassourinha & $x$ & $X$ \\
\hline Malvaceae & Whalteria indica & Falsa guanxuma & $x$ & - \\
\hline Poacea & Acanthospermum australe & Carrapichinho & $x$ & $\mathrm{X}$ \\
\hline Poacea & Eleusine indica & Pé de galinha & $x$ & $\mathrm{X}$ \\
\hline Solanaceae & Nicandra physaloides & Joá de capote & $\mathrm{X}$ & - \\
\hline Poacea & Pennisetum setosum & Capim custódio & $x$ & $\mathrm{X}$ \\
\hline Poacea & Setaria vulpiseta & Rabo de raposa & - & $\mathrm{X}$ \\
\hline Poacea & Uruchloa decumbens & Braquiária & $\mathrm{X}$ & - \\
\hline
\end{tabular}

As famílias que apresentaram maior número de espécies na área foram Asteraceae com seis espécies, Poaceae com cinco espécies e Malvaceae com quatro espécies. Essas três famílias totalizaram $60 \%$ das espécies encontradas. Asteraceae e Poaceae realmente são duas das principais famílias de plantas espontâneas existentes no Brasil (ADEGAS et al. 2010), pois, além de estarem presentes em áreas tradicionais de produção de grãos, também aparecem com grande importância em outros sistemas diferenciados de produção, como o da cana-de-açúcar (OLIVEIRA e FREITAS, 2008), de explorações de várzeas (SANTOS et al., 2004) e até em áreas de gramados (MACIEL et al., 2008).

Na fazenda Corinalves, nos anos de 2017 a 2019, em que foram identificadas 15 espécies distribuídas em 8 famílias Tabela 1. Novamente, as famílias que apresentaram maior número de espécies na área foram Poaceae com quatro espécies e Asteraceae com três espécies. Em levantamento fitossociológico realizado em cafezal orgânico no município de Garça (SP), Maciel et al (2010) também encontraram predominância dessas duas famílias em todas as variedades de café estudadas.

Na fazenda Piracanjuba, no ano de 2016, as principais espécies encontradas (IVC acima de 10\%) foram: Acanthospermum australe, Commelina benghalensis, Eleusine indica, Euphorbia hirta, Melampodium perfoliatum, Pennisetum setosum, Sida glaziovii e Uruchloa decumbens. a espécie Melampodium perfoliatum teve alta concentração de indivíduos em apenas uma área específica, caracterizando infestação do tipo reboleira (KISSMANN e GROTH, 1999), o que resultou em uma Frequência baixa, mas Densidade alta, resultando em um IVC também alto. Espécies com esse tipo de comportamento geralmente não causam grandes problemas, pois não há grande dificuldade para seu controle (Tabela 2). 
Tabela 2. Frequência $\left(F_{a n o}\right)$, Densidade $\left(D_{a n o}\right)$ e Índice de Valor de Cobertura $\left(I V C_{a n o}\right)$ das espécies avaliadas na fazenda Piracanjuba nos anos de 2016 a 2018. Silvânia, GO.

\begin{tabular}{|c|c|c|c|c|c|c|c|c|c|c|}
\hline Espécie & $\mathrm{F}_{16}$ & $\mathrm{~F}_{17}$ & $\mathrm{~F}_{18}$ & $\mathrm{D}_{16}$ & $\mathrm{D}_{17}$ & $\mathrm{D}_{18}$ & $\mathrm{IVC}_{16}$ & $\mathrm{IVC}_{17}$ & $\mathrm{IVC}_{18}$ & $\sum \mathrm{IVC}$ \\
\hline Pennisetum setosum & 0,9 & 1,0 & 1,0 & 13,3 & 28,1 & 507,2 & 31,5 & 29,4 & 105,7 & 166,6 \\
\hline $\begin{array}{l}\text { Alternanthera tenella } \\
\text { Melampodium }\end{array}$ & - & 1,0 & 0,6 & - & 74,4 & 1,6 & - & 62,2 & 6,8 & 69,0 \\
\hline $\begin{array}{l}\text { perfoliatum } \\
\text { Acanthospermum }\end{array}$ & 0,1 & - & - & 35,0 & - & - & 50,8 & - & - & 50,8 \\
\hline australe & 0,6 & 1,0 & 0,4 & 1,7 & 20,4 & 1,8 & 10,9 & 24,0 & 4,7 & 39,5 \\
\hline Commelina benghalensis & 0,1 & 1,0 & 0,6 & 6,0 & 4,8 & 2,4 & 9,9 & 12,9 & 7,0 & 29,8 \\
\hline Uruchloa decumbens & 1,0 & 0,4 & 0,2 & 3,1 & 0,5 & 0,6 & 18,5 & 4,2 & 2,3 & 24,9 \\
\hline Eleusine indica & 0,5 & 0,9 & - & 3,7 & 2,8 & - & 12,3 & 10,6 & - & 22,8 \\
\hline Sida rhombifolia & 0,5 & 0,4 & 0,8 & 1,5 & 0,5 & 2,2 & 9,2 & 4,2 & 9,1 & 22,4 \\
\hline Chamaesyce hirta & 0,6 & - & 0,6 & 2,5 & - & 5,2 & 12,0 & - & 7,5 & 19,5 \\
\hline Euphorbia heterophylla & 0,6 & 0,5 & 0,4 & 0,4 & 0,8 & 0,5 & 9,0 & 5,3 & 4,4 & 18,8 \\
\hline Emilia fosbergii & 0,3 & 0,6 & 0,6 & 0,7 & 1,3 & 1,0 & 5,2 & 6,6 & 6,7 & 18,6 \\
\hline Cyperus rotundus & 0,3 & 0,7 & 0,4 & 0,1 & 1,7 & 0,9 & 4,4 & 7,9 & 4,5 & 16,8 \\
\hline Sida glaziovii & 0,6 & 0,5 & - & 1,3 & 0,7 & - & 10,3 & 5,3 & - & 15,5 \\
\hline Amaranthus deflexus & 0,4 & 0,6 & - & 0,5 & 1,0 & - & 6,3 & 6,4 & - & 12,8 \\
\hline Ageratum conyzoides & 0,1 & - & 0,8 & 0,4 & - & 6,6 & 2,0 & - & 9,9 & 11,9 \\
\hline Ipomea triloba & 0,2 & 0,5 & 0,2 & 0,2 & 0,7 & 0,6 & 3,1 & 5,3 & 2,3 & 10,6 \\
\hline Bidens pilosa & 0,3 & - & 0,4 & 0,4 & - & 0,8 & 4,8 & - & 4,5 & 9,3 \\
\hline Conyza bonariensis & - & - & 0,6 & - & - & 1,1 & - & - & 6,7 & 6,7 \\
\hline Sida cordifolia & - & - & 0,6 & - & - & 0,8 & - & - & 6,7 & 6,7 \\
\hline Hyptis suaveolens & - & - & 0,6 & - & - & 0,6 & - & - & 6,6 & 6,6 \\
\hline Ricinus communis & - & 0,5 & 0 & - & 2,4 & 0 & - & 6,5 & 0 & 6,5 \\
\hline Whalteria indica & - & 0,5 & 0 & - & 0,6 & 0 & - & 5,2 & 0 & 5,2 \\
\hline Tridax procumbens & - & - & 0,4 & - & - & 1,1 & - & - & 4,6 & 4,6 \\
\hline Nicandra physaloides & - & 0,4 & - & - & 0,5 & - & - & 4,2 & - & 4,2 \\
\hline$\Sigma$ & 7,1 & 10,5 & 9,2 & 70,8 & 141,2 & 535 & - & - & - & - \\
\hline
\end{tabular}

No ano de 2017, as principais espécies encontradas foram Acanthospermum australe, Alternanthera tenella, Commelina benghalensis, Eleusine indica e Pennisetum setosum e, em 2018, Ageratum conyzoides, Pennisetum setosum e Sida rhombifolia (Tabela 2).

A espécie que merece maior destaque nos estudos realizados na fazenda Piracanjuba é a Pennisetum setosum que mostrou aumento significativo de infestação durante os três anos avaliados, o que pode ser corroborado pelo aumento de seu IVC observado na tabela 2. Segundo Lorenzi (2008), Essa é uma espécie da família das Poaceas, perene, de 80 a $180 \mathrm{~cm}$ de altura, nativa da América Tropical e sua propagação se dá por intermédio de sementes ou mesmo através de rizomas. É uma planta muito frequente em lavouras anuais e perenes, beira de estradas, carreadores e terrenos baldios, principalmente da região Centro-Oeste do país. É extremamente agressiva devido à grande produção de sementes que são dispersas, principalmente, pelo vento.

Na fazenda Corinalves, no ano de 2017, as principais espécies encontradas (IVC acima de 10\%) foram Alternanthera tenella, Commelina benghalensis, Ipomea triloba, Pennisetum setosum e Sida rhombifolia. No ano de 2018, as principais espécies foram Alternanthera tenella, Blainvillea rhomboidea, Commelina benghalensis, Eleusine indica, Ipomea triloba, Pennisetum setosum e Sida rhombifolia e, em 2019, Alternanthera tenella, Eleusine indica, Ipomea triloba, Pennisetum setosum e Sida rhombifolia (Tabela 3). 
Tabela 3. Frequência $\left(F_{a n o}\right)$, Densidade $\left(D_{a n o}\right)$ e Índice de Valor de Cobertura $\left(I V C_{a n o}\right)$ das espécies avaliadas na fazenda Corinalves nos anos de 2017 a 2019. Catalão, GO.

\begin{tabular}{|c|c|c|c|c|c|c|c|c|c|c|}
\hline Espécie & $\mathrm{F}_{17}$ & $\mathrm{~F}_{18}$ & $\mathrm{~F}_{19}$ & $D_{17}$ & $\mathrm{D}_{18}$ & $\mathrm{D}_{19}$ & $\mathrm{IVC}_{17}$ & $\mathrm{IVC}_{18}$ & $\mathrm{IVC}_{19}$ & $\Sigma \mathrm{IVC}$ \\
\hline Pennisetum setosum & 1,0 & 1,0 & 1,0 & 955,1 & 169,1 & 97,9 & 99,6 & 85,8 & 87,7 & 273,1 \\
\hline Alternanthera tenella & 1,0 & 0,9 & 1,0 & 31,4 & 5,3 & 32,2 & 16,5 & 14,9 & 43,1 & 74,6 \\
\hline Eleusine indica & - & 1,0 & 1,0 & - & 34 & 11,3 & 0,0 & 28,5 & 28,9 & 57,5 \\
\hline Ipomea triloba & 1,0 & 0,6 & 0,7 & 19,2 & 12,2 & 3,4 & 15,4 & 13,6 & 17,2 & 46,3 \\
\hline $\begin{array}{l}\text { Sida rhombifolia } \\
\text { Commelina }\end{array}$ & 0,6 & 0,9 & 0,5 & 23,3 & 3 & 1,0 & 10,3 & 13,9 & 11,3 & 35,6 \\
\hline benghalensis & 0,7 & 0,5 & 0,3 & 70,2 & 6,7 & 0,7 & 15,9 & 9,9 & 6,9 & 32,6 \\
\hline Blainvillea rhomboidea & 0,6 & 0,6 & 0,1 & 3,4 & 3,2 & 0,8 & 8,5 & 9,8 & 2,7 & 21,0 \\
\hline Emilia fosbergii & 0,3 & 0,4 & - & 0,4 & 0,5 & - & 4,1 & 5,8 & 0,0 & 10,0 \\
\hline Sida glaziovii & 0,6 & - & - & 5,8 & - & - & 8,7 & - & - & 8,7 \\
\hline $\begin{array}{l}\text { Setaria vulpiseta } \\
\text { Acanthospermum }\end{array}$ & 0,3 & 0,3 & - & 0,5 & 0,4 & - & 4,2 & 4,4 & - & 8,5 \\
\hline australe & - & 0,5 & - & - & 0,7 & - & - & 7,3 & - & 7,3 \\
\hline Senna ocidentalis & 0,5 & - & - & 1,2 & - & - & 7,0 & 0,0 & - & 7,0 \\
\hline Ageratum conyzoides & - & 0,4 & - & - & 0,6 & - & - & 5,9 & - & 5,9 \\
\hline Amaranthus reflexus & 0,4 & - & - & 1,1 & - & - & 5,6 & - & - & 5,6 \\
\hline Sebastiana corniculata & 0,3 & - & - & 0,6 & - & - & 4,2 & - & - & 4,2 \\
\hline Cyperus rothundus & - & - & 0,1 & - & - & 0,1 & - & - & 2,2 & 2,2 \\
\hline$\Sigma$ & 7,3 & 7,1 & 4,7 & 1112,2 & 235,7 & 147,4 & - & - & - & - \\
\hline
\end{tabular}

Nesse estudo fica evidente, observando-se principalmente os dados de Densidade, que houve acentuada redução na infestação da área de 2017 para 2019. Além da redução do número de espécies observadas, todas espécies que tiveram ocorrência nos três anos estudados, com exceção de Alternanthera tenella, tiveram sua população reduzida. Para essa área a sugestão é que se dê continuidade ao estudo fitossociológico nos próximos anos para verificar se há correlação entre a redução da infestação e o sistema de produção, uma vez que as comunidades espontâneas podem variar sua composição florística em função do tipo de manejo aplicado à área (ERASMO et al., 2004). O que se preconiza com o sistema corredores agroecológicos é a maior cobertura de solo, rotações, consórcios e uso de espécies com múltiplas funções, entre elas o controle de espontâneas, seja por cobertura de solo, deposição de matéria seca ou mesmo algum efeito alelopático.

Na fazenda Corinalves, a espécie que também mais se destacou foi a Pennisetum setosum, que apresentou elevados valores de Frequência e Densidade resultando em um IVC muito acima daquele apresentado pelas outras espécies, como pode ser observado na tabela 3. Essa espécie tem causado preocupação aos produtores da região, principalmente devido à grande dificuldade encontrada no seu manejo em função de sua agressividade, baseada na eficiência de produzir e dispersar sementes.

Segundo Carvalho e Malaquias (2019), espécies da família Poaceae são, relativamente, simples de serem controladas em sistemas convencionais devido à sua susceptibilidade à herbicidas, principalmente ao glifosato, sendo utilizadas, inclusive, em consórcio com outras culturas, visando o controle de espontâneas e a melhoria das condições do solo, como é o caso da braquiária. Já em sistemas orgânicos, dada a impossibilidade de utilização de herbicidas, tornam-se problema relevante, devido principalmente à sua capacidade de rebrota quando roçadas ou capinadas.

A continuidade do monitoramento e o estudo de medidas de controle, associando o uso de adubos verdes com roçadas e capina seletiva visando à redução da infestação de Pennisetum setosum, torna-se relevante para as duas áreas estudadas. Como pode-se observar através dos índices de valor de cobertura da espécie nas duas áreas onde ocorreu o estudo, não se trata de um problema localizado, mas de toda uma região, principalmente para produtores inseridos em sistemas orgânicos/agroecológicos, que têm seu custo de produção aumentado pela maior demanda de mão-deobra para controle de espontâneas.

O Coeficiente de Similaridade (CS) para os anos de 2017 e 2018 (57\%) mostrou similaridade mediana para a flora espontânea existente nas duas regiões (Tabela 4). 
Tabela 4 - Coeficientes de Similaridade entre as fazendas Piracanjuba (Pira) e Corinalves (Cori) nos anos de 2016 a 2019. Catalão, GO.

\begin{tabular}{ccccccc}
\hline & Pira & Pira & Pira & Cori & Cori & Cori \\
- & $\mathbf{2 0 1 6}$ & $\mathbf{2 0 1 7}$ & $\mathbf{2 0 1 8}$ & $\mathbf{2 0 1 7}$ & $\mathbf{2 0 1 8}$ & $\mathbf{2 0 1 9}$ \\
\hline Pira/2016 & 1 & 0,75 & 0,73 & - & - & - \\
Pira/2017 & - & 1 & 0,61 & - & 0,57 & - \\
Pira/2018 & - & - & 1 & - & - & - \\
Cori/2017 & - & - & - & 1 & 0,70 & 0,6 \\
Cori/2018 & - & - & - & - & 1 & 0,74 \\
Cori/2019 & - & - & - & - & - & 1 \\
\hline
\end{tabular}

A semelhança não pode ser atribuída à proximidade entre as fazendas, pois estão localizadas em diferentes municípios que apresentam considerável distância. Segundo Carvalho e Pitelli (1992), o fato de diferentes áreas apresentarem esse valor de coeficiente de similaridade, com mais da metade de espécies comuns, pode ser atribuído, talvez, às formas de manejo semelhantes entre essas áreas, o que propicia o desenvolvimento de determinadas espécies, em detrimento de outras.

Os índices de CS para a fazenda Piracanjuba indicam que a maioria das espécies são estáveis e foram encontradas nos três anos de estudo. Para a fazenda Corinalves, os índices de CS corroboram a afirmativa de que houve redução do número de espécies na área estudada de 2017 para 2019.

\section{Conclusões}

As famílias que apresentam maior número de espécies em ambas as áreas estudadas são Asteraceae e Poaceae. As espécies que apresentam maior relevância na fazenda Piracanjuba são: Acanthospermum australe, Commelina benghalensis, Eleusine indica e Pennisetum setosum e na fazenda Corinalves são: Alternanthera tenella, Commelina benghalensis, Eleusine indica, Ipomea triloba, Pennisetum setosum e Sida rhombifolia. A espécie que mais se destaca nas duas propriedades, com altos índices de Frequência, Densidade e Valor de Cobertura é Pennisetum setosum.

\section{Referências}

ADEGAS, F. S.; et al. Levantamento fitossociológico de plantas daninhas na cultura do girassol. Planta Daninha, v. 28, n. 4, p. 705-716, 2010.

BLACANCEAUX, P.; et al. Sistemas Pedológicos no Cerrado de Goiás: Município de Silvânia, Região Centro Oeste do Brasil. Rio de Janeiro, R.J.: Embrapa Solos, 2007. 90p. (Boletim de Pesquisa e Desenvolvimento, 117).

BRAUN-BLANQUET, J. Fitossociologia: bases para el estudio de las comunidades vegetales. Madrid: H. Blume, 1979. $820 \mathrm{p}$.

CARDOSO, A. D.; et al. Levantamento fitossociológico de plantas daninhas na cultura da mandioca em vitória da conquista, Bahia. Bioscience Journal, v. 29, n. 5, p. 1130-1140, 2013.

CARVALHO, S. L.; PITELLI, R. A. Levantamento e análise fitossociológica das principais espécies de plantas daninhas de pastagens da região de Selvíria (MS). Planta Daninha, v. 10, n. 1/2, p. 25-32, 1992.

CARVALHO, W. P.; MALAQUIAS, J. V. Plantas de cobertura para proteção do solo em sistema orgânico no período da seca na Região dos Cerrados. Planaltina, DF: Embrapa Cerrados, 2019. 27 p. (Embrapa Cerrados. Boletim de Pesquisa e Desenvolvimento, 347).

CHAVES, J. C. D.; CALEGARI, A. Adubação verde e rotação de culturas. Informe Agropecuário, v. 22, n. 212, p. 5360, 2001.

COSTA, P. F.; et al. Levantamento fitossociológico de plantas daninhas em latossolo cultivado com diferentes culturas de inverno em função dos manejos químico e mecânico. Cultivando o Saber, v. 7, n. 2, p. 192 - 204, 2014.

ERASMO, E. A. L.; et al. Levantamento fitossociológico das comunidades de plantas espontâneas em áreas de produção de arroz irrigado cultivado sob diferentes sistemas de manejo. Planta Daninha, v. 22, n. 2, p. 195-201, 2004.

FERREIRA, E. A.; et al. Levantamento fitossociológico de plantas daninhas em pastagens degradadas do Médio Vale do Rio Doce, Minas Gerais. Revista Ceres, v. 61, n. 4, p. 502-510, 2014. 
INSTITUTO MAURO BORGES DE ESTATÍSTICAS E ESTUDOS SOCIOECONÔMICOS (IMBEES) - Painéis Municipais, Catalão, 2016. 4p. > Disponível em: <www.imb.go.gov.br/files/docs/publicações/painéis-municipais/catalão> Acesso em: 02 mai. 2020.

KISSMANN, K. G.; GROTH, D. Plantas espontâneas e nocivas. 2.ed. São Paulo: BASF, 1999. 978 p.

LORENZI, H. Plantas daninhas do Brasil: terrestres, aquáticas, parasitas e tóxicas. 4. ed. Nova Odessa: Plantarum, 2008. 640p.

MACHADO, A. T.; MACHADO, C. T. T. Agrobiodiversidade e Corredores Agroecológicos. In: SANTILLI, J.; BUSTAMANTE, P.G.; BARBIERI, R.L. (eds): Coleção Transição Agroecológica: Agrobiodiversidade. Brasília: Embrapa, ABA, 2015. p. 103-124.

MACIEL, C. D. G.; et al. Composição florística da comunidade espontânea em gramados de Paspalum notatum no município de Assis, SP. Planta Daninha, v. 26, n. 1, p. 57-64, 2008.

MACIEL, C. D. G.; et al. Levantamento fitossociológico de plantas daninhas em cafezal orgânico. Bragantia, v. 69, n. 3, p. 631- 636, 2010.

MELO, A. V.; et al. Dinâmica populacional de plantas daninhas em cultivo de milho-verde nos sistemas orgânico e tradicional. Planta Daninha, v. 25, n. 3, p. 521-527, 2007.

MUELLER-DOMBOIS, D.; ELLENBERG, H. Aims and methods of vegetation ecology. New York: John Wiley \& Sons, 1974. 547 p.

OLIVEIRA, A. R.; FREITAS, S. P. Levantamento fitossociológico de plantas daninhas em áreas de produção de canade-açúcar. Planta Daninha, v. 26, n. 1, p. 33-46, 2008.

RESENDE, M.J.G. Distribuição dos solos de Goiás: Classes de solos dos municípios goianos. Goiânia: EMATER Agência Goiana de Assistência Técnica, Extensão Rural e Pesquisa Agropecuária. 2016, 57p.

SANTOS, L. D. T.; et al. Levantamento fitossociológico em pastagens degradadas sob condições de várzea. Planta Daninha, v. 22, n. 3, p. 343-349, 2004.

SILVA, F. J. C.; et al. Avaliação de índices fitossociológicos de plantas daninhas em solos com três diferentes texturas na cultura da cana-de-açúcar. Revista Fafibe On-Line, v. 10, n. 1, p. 223-240, 2017.

SORENSEN, T. A. Method of stablishing groups equal amplitude in plant society based on similarity of species content. In: ODUM, E.P. Ecologia. 3.ed. México: Interamericana, 1972. p. 341-405.

SOUZA, V. C.; LORENZI, H. Botânica sistemática: guia ilustrado para identificação das famílias de angiospermas da flora brasileira, baseado em APG II. 1a ed. Nova Odessa: Plantarum, 2005. 640p.

TAVARES, C. J.; et al. Fitossociologia de plantas daninhas na cultura do feijão. Revista Brasileira de Ciências Agrárias, v. 8, n. 1, p. 27-32, 2013 